\title{
The new classifications for thymic epithelial tumors: benefits and problems
}

\author{
Takayuki Fukui, Kohei Yokoi
}

Department of Thoracic Surgery, Nagoya University Graduate School of Medicine, Nagoya, Japan

Correspondence to: Takayuki Fukui, MD. Department of Thoracic Surgery, Nagoya University Graduate School of Medicine, 65 Tsurumai-cho, Showa-ku, Nagoya 466-8550, Japan. Email: tfukui@med.nagoya-u.ac.jp.

Provenance: This is an invited Editorial commissioned by Section Editor Dr. Gang Shen (The Second Affiliated Hospital Zhejiang University School of Medicine, Hangzhou, China).

Comment on: Meurgey A, Girard N, Merveilleux du Vignaux C, et al. Assessment of the ITMIG Statement on the WHO Histological Classification and of the Eighth TNM Staging of Thymic Epithelial Tumors of a Series of 188 Thymic Epithelial Tumors. J Thorac Oncol 2017;12:1571-81.

Submitted Sep 13, 2017. Accepted for publication Sep 22, 2017.

doi: $10.21037 /$ jtd.2017.09.119

View this article at: http://dx.doi.org/10.21037/jtd.2017.09.119

The tumor-node-metastasis (TNM) classification for cancer is a globally accepted principle used by oncologists. It provides planning treatments, indication of prognosis, evaluating the outcomes of treatments, facilitating the exchange of information between institutions, and facilitating the continued investigation of human cancers (1). For thymic epithelial tumors, the Masaoka classification was published in 1981 (2), and Koga et al. modified this classification in 1994 (3). This Masaoka-Koga classification has been widely used for a long time.

Recently, a new TNM classification for thymic epithelial tumors approved by both the Union for International Cancer Control and the American Joint Committee on Cancer was published as a part of the 8th edition of the TNM classification, proposed by the International Association for the Study of Lung Cancer (IASLC) and the International Thymic Malignancy Interest Group (ITMIG). The new classification was based on the large amount of data available in the global database created by the ITMIG, which consisted of 8,145 patients (4-8).

For the pathological diagnosis of thymic epithelial tumors, the World Health Organization (WHO) generated a classification that subdivides thymic epithelial tumors into $\mathrm{A}, \mathrm{AB}, \mathrm{B} 1, \mathrm{~B} 2$, and B3 (and other rare) thymomas and thymic carcinomas (9). However, the heterogeneity of the cellular components in these tumors contributes to the poor interobserver reproducibility in some studies (10-12). To overcome this problem, an ITMIG consensus statement for refining the criteria for each subcategory was published in 2014 (13).

\section{New TNM classification}

The T descriptor of the new TNM classification stated that tumors totally encapsulated, extend into the adjacent fat tissue, and invade the mediastinal pleura be defined collectively as T1 tumors. Only tumors that invade the pericardium are classified as $\mathrm{T} 2 . \mathrm{T} 3$ and $\mathrm{T} 4$ tumors invade lung, intrathoracic large vessels, and other tissues. Concerning the $\mathrm{N}$ descriptor, perithymic nodes were newly defined as $\mathrm{N} 1$ and deep intrathoracic/cervical nodes are defined as N2. Regarding the stage grouping, T1N0M0 was classified as stage I, T2N0M0 as stage II, T3N0M0 as stage IIIA, and T4N0M0 as stage IIIB. Tumors with positive N1 nodes and pleural dissemination were classified as stage IVA. Stage IVB tumors had lung nodules, N2 node involvement, and distant metastases.

Meurgey et al. assessed the new TNM classification and the ITMIG statement in a French cohort comprising 188 patients with thymic epithelial tumors (14). The breakdown of the patients by the new classification was as follows: stage I, $\mathrm{n}=127$ (83.6\%); stage II, $\mathrm{n}=3$ (2.0\%); stage III, $\mathrm{n}=17$ $(11.1 \%)$; stage IVA, $\mathrm{n}=2(1.3 \%)$, and stage IVB, $\mathrm{n}=3(2.0 \%)$ (Figure 1). In early 2016, we had already published the results of a clinical evaluation of the new TNM classification in 154 patients who underwent surgery in our institute (15), although it was not cited in the report by Meurgey et al. Using the new TNM system, our patients were reclassified as follows: stage I, $\mathrm{n}=119(77.3 \%)$; stage II, $\mathrm{n}=4(2.6 \%)$; stage IIIA, $\mathrm{n}=17(11.0 \%)$; stage IIIB, 


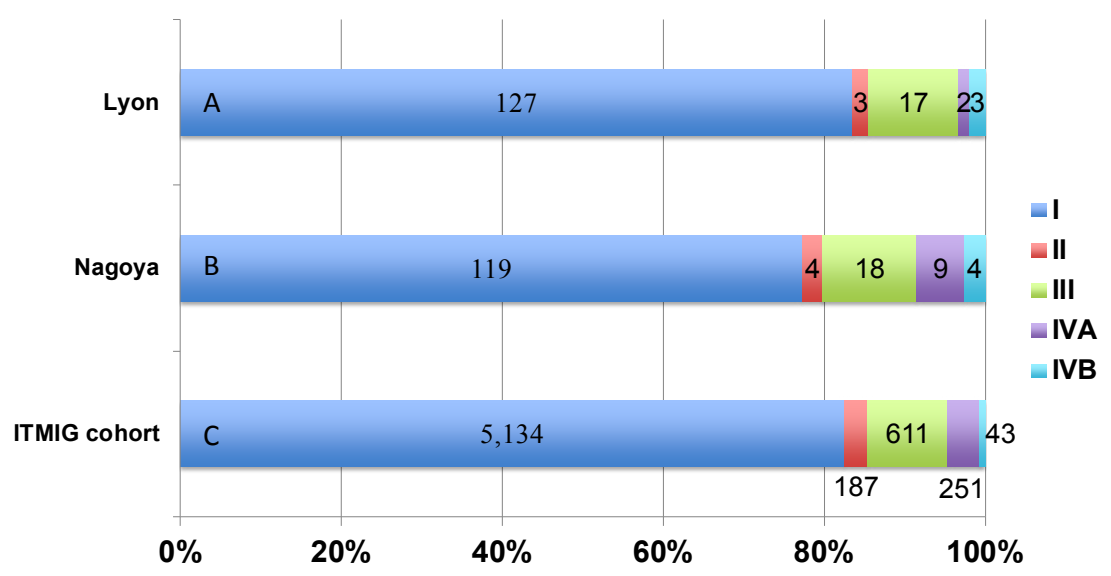

Figure 1 Distribution of the tumors according to the new TNM stage groupings. The actual number of tumors classified is represented by each bar. (A) Results of the French cohort reported by Meurgey et al. (14); (B) results of our cohort (15); (C) results using the ITMIG database (6). The proportion of patients with stage I tumors in each cohort was approximately $80 \%$.

$\mathrm{n}=1(0.6 \%)$; stage IVA, $\mathrm{n}=9(5.8 \%)$, and stage IVB, $\mathrm{n}=4$ (2.6\%) (Figure 1). In addition, a worldwide database created by the ITMIG (with 6,226 patients available) revealed the tumor distribution as follows: stage I, $82.5 \%$; stage II, $3.0 \%$; stage IIIA, $10.1 \%$; stage IIIB, $0.4 \%$; stage IVA, $4.0 \%$, and stage IVB, $0.7 \%$ (Figure 1) (6). These results were quite similar to those of the French cohort and our cohort. The proportion of patients with new stage I disease was remarkably increased because a part of patients with stage II and III disease of Masaoka-Koga system were reclassified and removed. Conversely, only a few patients were classified as having stage II tumors. In other words, the imbalance of the distribution was a result of disregarding whether a tumor was encapsulated or not in the defined new $\mathrm{T}$ descriptors. Detterbeck et al. suggested that whether the tumor was encapsulated did not have any prognostic impact, while mediastinal pleural involvement was an additional testing factor (6).

Survival analyses performed by Meurgey et al. did not show significant correlation between the new TNM staging and overall survival (OS) (14), similar to the report using the ITMIG database (6). However, time to recurrence, which was equal to recurrence-free survival (RFS), showed significant deterioration with increasing stage. Generally, recurrence is regarded as the best measure of outcome, especially for thymomas, which are rarely lethal and progress slowly. In fact, only eight patients died of thymic epithelial tumors in the French cohort. While generating the new TNM classification, each descriptor and stage groupings seemed to be based mainly on patient OS, i.e., on the concept of "prognostic grouping" in the recent trend. However, we believe that OS is complex and is influenced by many factors including age, sex, comorbidities, and social background. Therefore, stage grouping, which may accurately reflect recurrences, seems to be much more appropriate for thymic epithelial tumors.

\section{Pathological diagnosis}

The pathological diagnosis of thymic epithelial tumors according to the current WHO classification has been widely recognized to be variable owing to the heterogeneity of the various cellular components of the tumor (10-12). In the manuscript by Sakakura et al., a high rate of discordance in diagnosing thymic epithelial tumors between an expert pathologist of the thymus and a trained general pathologist was reported (11). The overall concordance rate was $63 \%$, in which 26 discordant cases of type $\mathrm{AB}$ thymoma were mainly diagnosed as type B1 or B2 by the general pathologist. Ten discordant cases of type B2 thymoma were diagnosed as type $\mathrm{AB}, \mathrm{B} 1$, or $\mathrm{B} 3$, and eight discordant cases of type B3 thymoma were diagnosed as type A, B2, or carcinoma. In addition, eight discordant cases of thymic carcinoma were diagnosed as type A or B3 thymoma. Verghese et al. also reported the reproducibility of the WHO classification of thymic epithelial tumors, in which distinguishing B1 thymomas from B2 and B2 thymomas from $B 3$ was determined to be difficult (12).

To overcome these problems to the maximum extent possible, the ITMIG consensus statement proposed major 
and minor morphological and immunohistochemical criteria to better individualize each subtype of thymic epithelial tumor (13). Consensus was achieved on refined criteria for decision-making regarding the $\mathrm{A} / \mathrm{AB}$ border; the distinction between B1, B2, and B3 thymomas; and the separation of B3 thymomas from carcinomas. In a cohort of 177 patients diagnosed in their institute, Meurgey et al. performed pathological reevaluation according to the ITMIG consensus major and minor criteria (14). The ITMIG consensus major criteria were identified in $100 \%$ of type A, $\mathrm{AB}, \mathrm{B} 1$, and $\mathrm{B} 2$ thymomas and $87 \%$ of thymic carcinomas. However, the minor criteria were not consistently observed, as only a minority of the tumors presented with all the minor criteria: $0 \%$ of seven type A thymomas, $22 \%$ of 67 type $\mathrm{AB}$ thymomas, $12 \%$ of 17 type $\mathrm{B} 1$ thymomas, $11 \%$ of 46 type $\mathrm{B} 2$ thymomas, and only $7 \%$ of thymic carcinomas. They stated that the value of the minor criteria might be debatable. To our knowledge, there has been no other study regarding the ITMIG consensus. However, the evaluation by Meurgey et al. was based on the assumption that the original diagnosis was accurate and did not consider that the ITMIG major and minor criteria would elicit a new diagnosis. Taken together, it still seemed to be not easy to obtain an accurate diagnosis of thymic epithelial tumors.

\section{Correlation between pathological diagnosis and TNM classification}

In the study by Meurgey et al., most type $\mathrm{A}, \mathrm{AB}$, and $\mathrm{B} 1$ thymomas were classified as stage I by the new TNM $(82 / 89$, $92 \%$ ) (14). Subsequently, the proportion of advanced tumors such as III and IV increased in the same order of type B2 and B3 thymoma and thymic carcinoma. In our analysis, among the 69 patients with type $\mathrm{A}, \mathrm{AB}$, or $\mathrm{B} 1$ thymoma, 68 (98\%) were re-classified as new stage I disease. Subsequently, the proportions of stage I were $55 \%$ and $38 \%$ in type B3 and thymic carcinoma, respectively (15). Hence, we believe the future of histopathologic classification of thymomas might require a simpler one. Previously, Suster et al. proposed a three-tiered classification. Briefly, type $A, A B$, $\mathrm{B} 1$, and $\mathrm{B} 2$ thymoma of the WHO classification collectively grouped as well-differentiated thymic carcinoma, type B3 thymoma and the current "thymic carcinoma" was renamed as moderately differentiated thymic carcinoma and poorly differentiated carcinoma, respectively (16). The findings by Meurgey et al. as well as our study findings support this proposed terminology.

\section{Benefits and problems}

The development of a new TNM classification for thymic malignancies can be a very useful tool for thoracic clinicians. The newly defined TNM classification appears to be functional and worthwhile, especially in clinical settings and RFS analysis. However, it did bring the significant imbalances of stage distribution and the new stage grouping could not serve as a predictor of OS.

The most significant limitation of the retrospective study by Meurgey et al. on thymic epithelial tumors was the small number of patients for analysis. The current TNM system developed by IASLC and ITMIG was based on the record of over 8,000 patients from the globally collected database. In addition, the postoperative follow-up period in both French cohort and ours might be relatively short for thymic malignancies, because recurrence beyond 5 years after surgery is not uncommon. Thus, there seems to be still room for survival analyses including OS and RFS of the patients with thymic epithelial tumors.

Ideally, further validation of this new TNM classification and a detailed consensus of the pathological diagnosis for these rare malignancies using larger cohorts are warranted.

\section{Acknowledgements}

None.

\section{Footnote}

Conflicts of Interest: The authors have no conflicts of interest to declare.

\section{References}

1. Sobin LH, Gospodarowicz MK, Wittekind C. editors. TNM Classification of Malignant Tumours, 7th Edition. Wiley-Blackwell, 2011.

2. Masaoka A, Monden Y, Nakahara K, et al. Follow-up study of thymomas with special reference to their clinical stages. Cancer 1981;48:2485-92.

3. Koga K, Matsuno Y, Noguchi M, et al. A review of 79 thymomas: modification of staging system and reappraisal of conventional division into invasive and non-invasive thymoma. Pathol Int 1994;44:359-67.

4. Nicholson AG, Detterbeck FC, Marino M, et al. The IASLC/ITMIG Thymic Epithelial Tumors Staging Project: proposals for the T Component for the 
forthcoming (8th) edition of the TNM classification of malignant tumors. J Thorac Oncol 2014;9:S73-80.

5. Kondo K, Van Schil P, Detterbeck FC, et al. The IASLC/ITMIG Thymic Epithelial Tumors Staging Project: proposals for the $\mathrm{N}$ and $\mathrm{M}$ components for the forthcoming (8th) edition of the TNM classification of malignant tumors. J Thorac Oncol 2014;9:S81-7.

6. Detterbeck FC, Stratton K, Giroux D, et al. The IASLC/ ITMIG Thymic Epithelial Tumors Staging Project: proposal for an evidence-based stage classification system for the forthcoming (8th) edition of the TNM classification of malignant tumors. J Thorac Oncol 2014;9:S65-72.

7. Bhora FY, Chen DJ, Detterbeck FC, et al. The ITMIG/ IASLC Thymic Epithelial Tumors Staging Project: A Proposed Lymph Node Map for Thymic Epithelial Tumors in the Forthcoming 8th Edition of the TNM Classification of Malignant Tumors. J Thorac Oncol 2014;9:S88-96.

8. Detterbeck FC, Asamura H, Crowley J, et al. The IASLC/ ITMIG thymic malignancies staging project: development of a stage classification for thymic malignancies. J Thorac Oncol 2013;8:1467-73.

9. Tumours of the Thymus. Available online: https://www. iarc.fr/en/publications/pdfs-online/pat-gen/bb10/bb10chap3.pdf

10. Wang H, Sima CS, Beasley MB, et al. Classification of thymic epithelial neoplasms is still a challenge to thoracic pathologists: a reproducibility study using digital microscopy. Arch Pathol Lab Med 2014;138:658-63.

11. Sakakura N, Tateyama H, Nakamura S, et al. Diagnostic reproducibility of thymic epithelial tumors using the World Health Organization classification: note for thoracic clinicians. Gen Thorac Cardiovasc Surg 2013;61:89-95.

12. Verghese ET, den Bakker MA, Campbell A, et al. Interobserver variation in the classification of thymic tumours--a multicentre study using the WHO classification system. Histopathology 2008;53:218-23.

13. Marx A, Ströbel P, Badve SS, et al. ITMIG consensus statement on the use of the WHO histological classification of thymoma and thymic carcinoma: refined definitions, histological criteria, and reporting. J Thorac Oncol 2014;9:596-611.

14. Meurgey A, Girard N, Merveilleux du Vignaux C, et al. Assessment of the ITMIG Statement on the WHO Histological Classification and of the Eighth TNM Staging of Thymic Epithelial Tumors of a Series of 188 Thymic Epithelial Tumors. J Thorac Oncol 2017;12:1571-81.

15. Fukui T, Fukumoto K, Okasaka T, et al. Clinical evaluation of a new tumour-node-metastasis staging system for thymic malignancies proposed by the International Association for the Study of Lung Cancer Staging and Prognostic Factors Committee and the International Thymic Malignancy Interest Group. Eur J Cardiothorac Surg 2016;49:574-9.

16. Suster S, Moran CA. Thymoma classification: current status and future trends. Am J Clin Pathol 2006;125:542-54.
Cite this article as: Fukui T, Yokoi K. The new classifications for thymic epithelial tumors: benefits and problems. J Thorac Dis 2017;9(11):4165-4168. doi: 10.21037/jtd.2017.09.119 